\title{
Multi-step inertial proximal contraction algorithms for monotone variational inclusion problems
}

\section{CUIJIE ZHANG, QIAO-LI DONG and JIAJIA CHEN}

\begin{abstract}
.
In this article, we introduce the multi-step inertial proximal contraction algorithms (MiPCA) to approximate a zero of the sum of two monotone operators, with one of the two operators being monotone and Lipschitz continuous. The weak convergence of the MiPCA is shown under the summability condition formulated in terms of the iterative sequence in a Hilbert space setting. We also investigate the unconditional convergence of the one-step inertial proximal contraction algorithm. Finally, numerical experiments are given to illustrate the advantage of the multi-step inertial proximal contraction algorithms.
\end{abstract}

Acknowledgements. The authors would like to thank the anonymous reviewer for the comments on the manuscript which helped us very much in improving and presenting the original version of this paper.

The first author is supported by Scientific Research Project of Tianjin Municipal Education Commission (No: 2018KJ253).

\section{REFERENCES}

[1] Bauschke, H. H., Bui, M. N. and Wang, X., Applying FISTA to optimization problems (with or) without minimizers, Math. Program., https://doi.org/10.1007/s10107-019-01415-x, (2019)

[2] Bauschke, H. H. and Combettes, P. L., Convex Analysis and Monotone Operator Theory in Hilbert Spaces, Second Edition, Springer, 2017

[3] Beck, A. and Teboulle, M., A fast iterative shrinkage-thresholding algorithm for linear inverse problems, SIAM J. Imaging Sci., 2 (2009), 183-202

[4] Brézis, H., Opérateur maximaux monotones, In Mathematics Studies, 1973

[5] Ceng, L. C., Lin, Y. C. and Wen, C. F., Iterative methods for triple hierarchical variational inequalities with mixed equilibrium problems, variational inclusions, and variational inequalities constraints, J. Inequal. Appl., 2015 2015:16, 62 pp.

[6] Chambolle, A. and Dossal, Ch., On the Convergence of the Iterates of the Fast Iterative Shrinkage/ Thresholding Algorithm, J. Optim. Theory Appl., 166 (2015), 968-982

[7] Dong Q. L., Cho Y. J., Zhong L. L. and Rassias T. M., Inertial projection and contraction algorithms for variational inequalities, J. Global Optim., 70 (2018), 687-704

[8] Dong, Q. L., Huang, J., Li, X. H., Cho, Y. J. and Rassias, Th. M., MiKM: Multi-step inertial Krasnosel'skiǔMann algorithm and its applications, J. Global Optim., 73 (2019), No. 4, 801-824

[9] Dong, Q. L., Jiang, D., Cholamjiak, P. and Shehu, Y., A strong convergence result involving an inertial forward-backward algorithm for monotone inclusions, J. Fixed Point Theory Appl., 19 (2017), 3097-3118

[10] Dong, Q. L., Lu, Y. Y. and Yang, J., The extragradient algorithm with inertial effects for solving the variational inequality, Optim., 65 (2016), No. 12, 2217-2226

Received: 09.11.2019; In revised form: 31.01.2020; Accepted: 07.02.2020

2010 Mathematics Subject Classification. 90C47, 49J35.

Key words and phrases. monotone variational inclusion problem, resolvent operator, multi-step inertial proximal contraction algorithm, one-step inertial proximal contraction algorithm.

Corresponding author: Qiao-Li Dong; dongql@lsec.cc.ac.cn 
[11] Fan, J., Liu, L. and Qin, X., A subgradient extragradient algorithm with inertial effects for solving strongly pseudomonotone variational inequalities, Optimization, https://doi.org/10.1080/02331934.2019.1625355, (2020)

[12] Fang, Y. P. and Huang, N. J., H-monotone operator and resolvent operator technique for variational inclusion, Appl. Math. Comput., 145 (2006), 795-803

[13] Harker, P. T. and Pang, J. S., A damped-newton method for the linear complementarity problem, Lect. Appl. Math., 26 (1990), 265-284

[14] He, B. S., A class of projection and contraction methods for monotone variational inequalities, Appl. Math. Optim., 35 (1997), 69-76

[15] Hieu, D. V., Anh, P. K. and Muu, L. D., Modified hybrid projection methods for finding common solutions to variational inequality problems, Comput. Optim. Appl., 66 (2017), 75-96

[16] Huang, N. J., A new completely general class of variational inclusions with noncompact valued mappings, Comput. Math. Appl., 35 (1998), No. 10, 9-14

[17] Jitpeera, T. and Kumam, P., A new hybrid algorithm for a system of mixed equilibrium problems, fixed point problems for nonexpansive Semigroup, and variational inclusion problem, Fixed Point Theory Appl., 2011 (2011), 217407

[18] Liang, J. and Schönlieb, C. B., Improving FISTA: Faster, Smarter and Greedier, https: / /arxiv.org/abs/1811.01430v2, (2019)

[19] Liang, J., Convergence Rates of First-Order Operator Splitting Methods, Optim. Cont [math.OC]. Normandie Université. Greyc Cnrs Umr 6072, 2016

[20] Lions, P. L. and Mercier, B., Splitting algorithms for the sum of two nonlinear operators, SIAM J. Numer. Anal., 16 (1979), 964-979

[21] Lorenz, D. A. and Pock, T., An inertial forward-backward algorithm for monotone inclusions, J. Math. Imaging Vis., 51 (2015), 311-325

[22] Moudafi, A. and Oliny, M., Convergence of a splitting inertial proximal method for monotone operators, J. Comp. Appl. Math., 155 (2003), 447-454

[23] Nesterov, Y. E., A method for solving the convex programming problem with convergence rate $O\left(1 / k^{2}\right)$, Dokl. Akad. Nauk SSSR., 269 (1983), 543-547 (in Russian)

[24] O'Donoghue, B. and Candès, E. J., Adaptive restart for accelerated gradient schemes, Found. Comput. Math., 15 (2015), No. 3, 715-732

[25] Polyak, B. T., Introduction to Optimization, Optimization Software 1987

[26] Polyak, B. T., Some methods of speeding up the convergence of iteration methods, U.S.S.R. Comput. Math. Math. Phys., 4 (1964), No. 5, 1-17

[27] Rockafellar, R. T., Monotone operators and the proximal point algorithms, SIAM J. Control Optim., 14 (1976), No. 5, 877-898

[28] Shehu, Y. and Cholamjiak, P., Iterative method with inertial for variational inequalities in Hilbert spaces, CALCOLO., 56 (2019), No. 1, Art. 4, 21 pp.

[29] Shehu, Y., Dong, Q. L. and Jiang, D., Single projection method for pseudo-monotone variational inequality in Hilbert spaces, Optimization, 68 (2019), 385-409

[30] Tang, Y. C., Wu, G. R. and Zhu, C. X., A first-order splitting method for solving a large-scale composite convex optimization Problem, J. Comp. Math., 37 (2019), No. 5, 668-690

[31] Thong, D. V. and Hieu, D. V., Inertial subgradient extragradient algorithms with line-search process for solving variational inequality problems and fixed point problems, Numer. Algorithms, 80 (2019), 1283-1307

[32] Tibshirani, R., Regression Shrinkage and Selection Via the Lasso, J. Royal Stat. Soc., 58 (1996), 267-288

[33] Verma, R. U., A-monotone nonlinear relaxed cocoercive variational inclusions, Cent. Eur. J. Math., 5 (2007), 386-396

[34] Verma, R. U., Approximation-solvability of a class of A-monotone variational inclusion problems, J. Korea Soc. Indus. Appl Math., 8 (2004), No. 1, 55-66

[35] Zeng, L. C., Guu, S. M. and Yao, J. C., Characterization of H-monotone operators with applications to variational inclusions, Comput. Math. Appl., 50 (2005), No. 3-4, 329-337

[36] Zhang, S. S., Lee, J. H. W. and Chan, C. K., Algorithms of common solutions to quasi variational inclusion and fixed point problems, Appl. Math. Mech., 29 (2008), 571-581

[37] Zhang, C. and Wang, Y., Proximal algorithm for solving monotone variational inclusion, Optimization, 67 (2018), No. 8, 1197-1209

[38] Zhao, J. and Yang, Q., Self-adaptive projection methods for the multiple-sets split feasibility problem, Inverse Probl., 27 (2011), No. 3, 035009, 13 pp. 
Civil AViation University OF CHinA

COLLEGE OF SCIENCE

TIANJIN, 300300, CHINA

Email address: c jzhang@cauc.edu.cn

Email address: dongql@ lsec.cc.ac.cn

Email address: $2428743880 @$ qq. com 